and shorter on the klinostat than in upright plants. On the klinostat there is less sclerenchyma formed. The walls of the sclerenchymatous cells are much thinner and the cells are half as long and more than twice as wide as in upright fronds. Heyn ${ }^{4}$ has shown that geotropic stimulus causes changes in the plasticity of cell walls as a result of auxin redistribution. In this case the continual transverse auxin supply prevents the cells ageing and losing their plasticity, and the formation of sclerenchyma is retarded. In all cases examined, no difference was found in the distribution of starch and statoliths in plants on the klinostat. Borgström questions the idea of the statolith mechanism and geotropic reaction being causally related. He considers that it is the transverse distribution of auxin caused by gravity which increases the mobility of the starch grains and facilitates their falling, at the same time inducing differential growth of longitudinal and transverse cell walls. From experiments with onion and oat roots he shows that auxin activates diastatic pro- cesses and accelerates the transformation of starch into sugar, and he assumes that it is a transverse distribution of auxin which causes the disappearance of starch from developing tissues, making sugar available for growth purposes. Should this be so, one would expect to find that an artificially induced transverse distribution would change the distribution of starch in klinostat plants.

It is clear that a change in the direction of auxin distribution may alter the plasticity of cell walls and lead to definite changes in plant tissues. The possible applications of this theory offer wide scope to the research worker in many problems of plant physiology and morphology. ${ }^{1}$ Borgström, Georg, "The Transverse Reactions of Plants : Outlines
of a New Interpretation of the Significance of Growth Hormones
for Life-Processes in Plants". Pp. 230. (Lund : C. W. K. Gleerup ;
Copenhagen: Ejnar Munksgaard; London: Williams and Nor-
gate, Ltd., 1939.) 6.00 kr.

2 Brain, E, D., New Phytol, 33, 3 (1939).

3 Zollikofer, C., Ber. deutsch. Bot. Ges., 53, 152-157 (1935)

‘ Heyn, A. N. J., Jb. wiss. Bot., 79, 753-787 (1934).

\title{
THE SCATTERING OF WAVES IN RADIO TRANSMISSION
}

$\mathrm{F}^{\mathrm{a}}$ OR many years past, the mode of propagation of radio waves through the ionosphere has been studied intensively by mathematicians, physicists and radio communication engineers. It is now well known that all long-distance communication takes place by deflection of the waves in one or other of the ionized regions of the atmosphere, and furthermore that, depending upon the density of ionization of any particular region, there is a maximum frequency of the waves which will be deflected and above which the waves penetrate the region and are not returned to the earth. This critical penetration frequency increases with the angle of incidence of the waves on the ionosphere. Thus, while the general features of long-distance radio transmission can be interpreted in terms of a simple ray treatment of the waves passing from the earth up to the ionosphere and back to the earth, there is a minimum distance from the sending station at which signals can be received by this mode of propagation. This 'skip' zone or distance, as it is termed, is naturally subject to diurnal and seasonal variations, as well as to the actual frequency used in the transmission.

Although all normal commercial communication takes place by the transmission of waves to ranges beyond the skip zone, it has been known for some time that, inside this zone, signals may be received more or less spasmodically, these signals being very irregular and subject to large variations in both intensity and direction of arrival at the receiving station. This type of reception has been interpreted as being due to the scattering of waves from local irregular portions or clouds in the ionosphere, where the intensity of ionization is such as to give rise to a diffuse reflection of the waves in all directions.

In a paper read before the Wireless Section of the Institution of Electrical Engineers on February 7, Mr. T. L. Eckersley presented an analysis of the effect of scattering in radio transmission. The earliest experimental evidence of scattering was obtained from observations at Chelmsford in 1927, when the shòrt-wave beam stations were first opened. In general, such scattered signals within the skip zone gave no definite bearing indication on a directionfinder unless the sending aerial emitted a directive beam, in which case the apparent direction of travel of the waves was approximately the opposite to that in which the beam of rays was projected. In later work, it has been possible to measure the time delay of the scattered signals in relation to the ground or direct ray, and also in some instances to the normal echoes received after one or two reflections from the $F$ region of the ionosphere. The scattered echoes are of relatively low intensity and thus only become suitable for quantitative observation when a highpower transmitter is available, and the use of a pulse modulation makes possible the measurement of the time of arrival of the individual echoes.

Photographic records demonstrated by $\mathrm{Mr}$. Eckersley showed that the scattered echoes have a moderately well-defined minimum time of arrival, but beyond this, the received echoes are spread over a considerable period in an irregular manner. A complete investigation of the phenomena showed that the cause of such scattering lies in momentary irregularities and small clouds in the $E$ region of the ionosphere. Since the wave frequencies employed were generally much greater than the critical frequency of the $E$ region, the path of transmission from the sender involved penetration of this region, reflection at the higher $F$ region, followed by scattering from the upper side of the $E$ region, and so by a second reflection from the $F$ region back to the receiving station on the ground. The time delays of the echoes fits in with this explanation, and the fact that the average intensity of the scattered signals varies approximately as the fourth power of the wavelength is in accordance with the scattering coefficient of ionic clouds in the $E$ region.

The effect of scattering is of major importance in the analysis of long-distance radio transmission phenomena, and the results of the investigation described in the paper referred to do much to increase our knowledge of the subject and our understanding of the mechanism by which the scattering is produced. 